\title{
Time series forecasting using Holt-Winters exponential smoothing: An application to economic data
}

Cite as: AIP Conference Proceedings 2186, 090003 (2019); https://doi.org/10.1063/1.5137999

Published Online: 10 December 2019

Susana Lima, A. Manuela Gonçalves, and Marco Costa

ARTICLES YOU MAY BE INTERESTED IN

Arima model and exponential smoothing method: A comparison

AIP Conference Proceedings 1522, 1312 (2013); https://doi.org/10.1063/1.4801282

Forecasting of primary energy consumption data in the United States: A comparison between ARIMA and Holter-Winters models

AIP Conference Proceedings 1885, 020163 (2017); https://doi.org/10.1063/1.5002357

Forecasting time series combining Holt-Winters and bootstrap approaches

AIP Conference Proceedings 1648, 110004 (2015); https://doi.org/10.1063/1.4912411

Challenge us.

What are your needs for periodic signal detection?

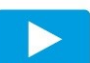

Watch

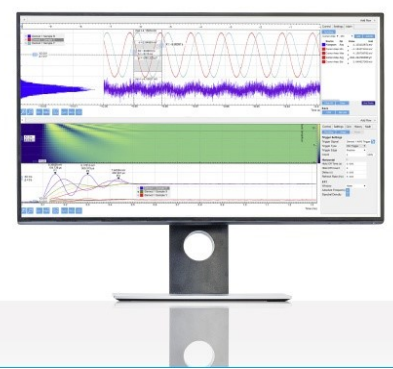

- Zurich Instruments 


\title{
Time Series Forecasting using Holt-Winters Exponential Smoothing: an Application to Economic Data
}

\author{
Susana Lima ${ }^{1, b)}$, A. Manuela Gonçalves ${ }^{2, a)}$ and Marco $\operatorname{Costa}^{3, c)}$ \\ ${ }^{1}$ Department of Mathematics, University of Minho, Portugal. \\ ${ }^{2}$ Department of Mathematics and Center of Mathematics, University of Minho, Portugal. \\ ${ }^{3}$ Águeda School of Technology and Management and Center for Research and Development in Mathematics and \\ Applications, University of Aveiro, Portugal.

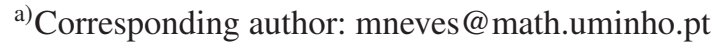

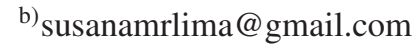 \\ ${ }^{c)}$ marco@ua.pt
}

\begin{abstract}
This study deals with forecasting economic time series that have strong trends and seasonal patterns. How to best model and forecast these patterns has been a long-standing issue of time series analysis. In this work, we propose a Holt-Winters Exponential Smoothing approach to time series forecasting in order to increase the chance of capturing different patterns in the data and thus improve forecasting performance. Therefore, the main propose of this study is to compare the accuracy of Holt-Winters models (additive and multiplicative) for forecasting and to bring new insights about the methods used via this approach. These methods are chosen because of their ability to model trend and seasonal fluctuations present in economic data. The models are fitted to time series of e-commerce retail sales in Portugal. Finally, a comparison is made and discussed.
\end{abstract}

\section{INTRODUCTION}

Time series forecasting is an important area of forecasting in which past observations of the same variable are collected and analyzed to develop a model describing the underlying relationship. The model is then used to extrapolate the time series into the future. Forecasting methods are a key tool in decision-making processes in many areas, such as economics, management and finance, or environment. Much effort has been devoted over the past several decades to the development and improvement of time series forecasting models. The main propose of this work is to compare the accuracy of the additive and the multiplicative Holt-Winters Exponential Smoothing models for forecasting economic time series; both models can increase the chance of capture different patterns in the data and improve forecasting performance.

According to the GfK study "European Retail 2016" that analyzes 33 countries in Europe, the retail segment in private consumption, in Portugal, in the year 2015, accounted for $33.4 \%$. The study of the economic variables associated with this area is essential and quite useful both to characterize the recent past and to anticipate trends. Obtaining empirical results from several real data sales forecasting is one of the most important issues behind all strategic and planning decisions in any retail business. The objective of the turnover index is to show the development of the market for goods and services. Turnover comprises the totals invoiced by the observation unit during the reference period, and this corresponds to market sales of goods or services supplied to third parties. Turnover also includes all other charges (transport, packaging, etc.) passed on to the customer, even if these charges are listed separately in the invoice. Turnover excludes VAT and other similar deductible taxes directly linked to turnover, as well as all duties and taxes on the goods or services invoiced by the unit. The data used in this study, available on the Eurostat platform, corresponds to monthly indexes of turnover (total, TOVT) in the context of retail sales via internet (e-commerce) observed between January 2000 and February 2018 (Fig. 1). Note that this index should be compared with the base year, in this case 2015, which correspond to the index 100.

\footnotetext{
Proceedings of the International Conference of Computational Methods in Sciences and Engineering 2019 (ICCMSE-2019) AIP Conf. Proc. 2186, 090003-1-090003-4; https://doi.org/10.1063/1.5137999 Published by AIP Publishing. 978-0-7354-1933-9/\$30.00
} 


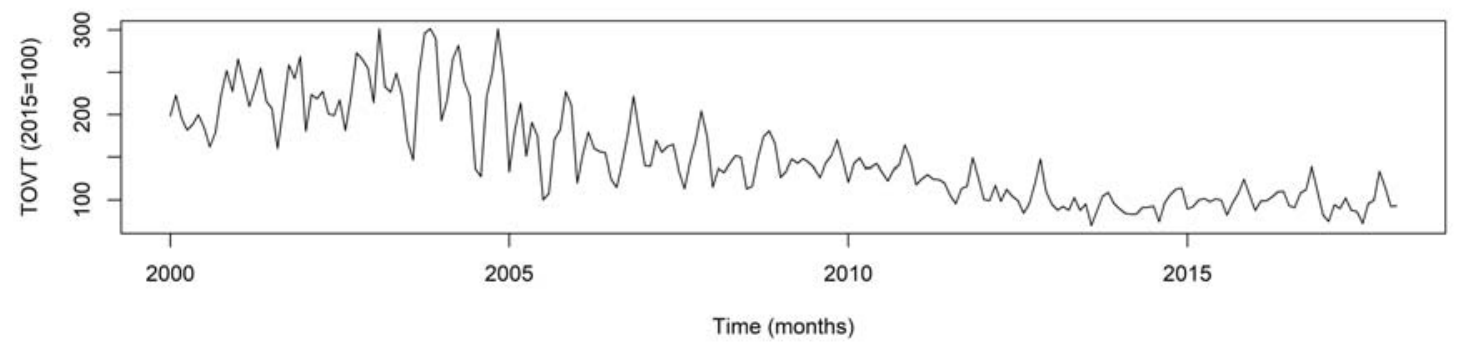

FIGURE 1. E-commerce retail sales time series of Portugal.

\section{METHODS}

The Holt-Winters (HW) method is an extension of the Holt method, and is applied whenever the data behaviour is trendy and is seasonal [2]. Relatively to the seasonal type, it can be additive or multiplicative, depending on the oscillatory movement along the time period. In both versions, forecasts will depend on the following three components of a seasonal time series: its level, its trend and its seasonal coefficient. In addition, both are implemented in the HoltWinters() function of the forecast package in $\mathrm{R}$ [3]. The additive version ought to be considered whenever the seasonal pattern of a series has a constant amplitude over time. In the additive case, the series can be written by $Y_{t}=T_{t}+S_{t}+\epsilon_{t}$, where $T_{t}$ represents the trend (the sum of the level and slope of the series at time $t$ ), $S_{t}$ is the seasonal component, and $\epsilon_{t}$ are error terms with mean zero and constant variance. In the multiplicative case, the series can be represented by $Y_{t}=T_{t} \times S_{t}+\epsilon_{t}$. The recursive equations of the multiplicative and additive HW methods, for level, trend, seasonal factors and forecast, with $h_{s}^{+}=[(h-1) \bmod s]+1$, are presented in Table 1 , where $Y_{t}$ is the observed data at time $t, s$ is the length of seasonality (number of months in a season), $h$ is the number of forecast ahead, and $\theta=(\alpha, \beta, \gamma)^{T}$ is the vector of smoothing parameters [4].

TABLE 1. Holt-Winters method recursive equations.

\begin{tabular}{l|l}
\hline Additive HW method & Multiplicative HW method \\
\hline Level: $l_{t}=\alpha\left(Y_{t}-s_{t-s}\right)+(1-\alpha)\left(l_{t-1}+b_{t-1}\right), 0 \leq \alpha \leq 1$ & Level: $l_{t}=\alpha \frac{Y_{t}}{s_{t-s}}+(1-\alpha)\left(l_{t-1}+b_{t-1}\right), 0 \leq \alpha \leq 1$ \\
Trend: $b_{t}=\beta\left(l_{t}-l_{t-1}\right)+(1-\beta) b_{t-1}, 0 \leq \beta \leq 1$ & Trend: $b_{t}=\beta\left(l_{t}-l_{t-1}\right)+(1-\beta) b_{t-1}, 0 \leq \beta \leq 1$ \\
Seasonal: $s_{t}=\gamma\left(Y_{t}-l_{t}\right)+(1-\gamma) s_{t-s}, 0 \leq \gamma \leq 1$ & Seasonal: $s_{t}=\gamma \frac{Y_{t}}{l_{t}}+(1-\gamma) s_{t-s}, 0 \leq \gamma \leq 1$ \\
Forecast: $\hat{Y}_{t+h}=l_{t}+h b_{t}+s_{t-s+h_{s}^{+}}, h=1,2, \ldots$ & Forecast: $\hat{Y}_{t+h}=\left(l_{t}+h b_{t}\right) s_{t-s+h_{s}^{+}}, h=1,2, \ldots$ \\
\hline
\end{tabular}

To initialize these methods, the $\mathrm{R}$ environment uses the decompose function to perform a decomposition of the series in its components - trend and seasonality- through a moving average process, and thus determine the initial values for $l_{s}, b_{s}$ and $s_{i}, i=1, \ldots, s\left(\hat{l}_{s} \mathrm{e} \hat{b}_{s}\right.$ are determined by means of the trend of a simple linear regression by using the initial observations).

Computing prediction intervals is an important part of the forecasting process, intended to indicate the likely uncertainty in point forecasts. The forecast intervals are usually based on the Mean Square Error (MSE) that denotes the variance of the $h$-step-ahead forecast errors. Empirical forecast intervals (if a normality assumption is verified) at $h$-step-ahead and the time series presents a seasonal component with period $s$ are given by the following expression

$$
\left(\hat{Y}_{t+h}-z_{1-\alpha / 2} \sqrt{\mathrm{MSE}_{(h)}}, \hat{Y}_{t+h}+z_{1-\alpha / 2} \sqrt{\mathrm{MSE}_{(h)}}\right),
$$

where $z$ is the appropriate quantile for the standard Normal distribution, and $\operatorname{MSE}_{(h)}=\frac{1}{n-h-s+1} \sum_{t=h+s}^{n}\left(Y_{t}-\hat{Y}_{t \mid t-h}\right)^{2}$ denotes the variance of the $h$-step-ahead errors. In order to compare the predictive accuracy of both methods, we considered five forecasting performance criteria: Mean Square Error (MSE), Root Mean Square Error (RMSE), Mean Absolute Percentage Error (MAPE), Mean Absolute Scaled Error (MASE), and Theil's U-statistics. 


\section{RESULTS AND DISCUSSION}

The methods considered in this study are applied to two sets: training data (in-sample data) and testing data (outof-sample data) in order to testify the accuracy of the proposed forecasting models. The selected training data from January 2000 to December 2016 (first 204 months) was used in order to fit the models to the data, and the test period from January 20017 to February 2018 (last 14 months) was used to forecast. The smoothing constants estimates and the values used for the initialization of both multiplicative and additive methods can be found in Table 2 and Table 3 , respectively.

TABLE 2. Initialization of level, trend, seasonal and exponential smoothing parameters of the multiplicative HW model for the e-commerce retail sales time series of Portugal.

\begin{tabular}{lllcc}
\hline Multiplicative model & \multicolumn{3}{c}{ MSE $=420.3237$} \\
\hline$\hat{\alpha}=0.1823$ & $\hat{\beta}=0.0589$ & $\hat{\gamma}=0.6781$ & $\hat{l}_{1}=203.2920$ & $\hat{b}_{1}=2.2286$ \\
$\hat{s}_{1}=1.1915$ & $\hat{s}_{2}=1.0632$ & $\hat{s}_{3}=0.9320$ & $\hat{s}_{4}=1.0118$ & $\hat{s}_{5}=1.1139$ \\
$\hat{s}_{6}=0.9367$ & $\hat{s}_{7}=0.8972$ & $\hat{s}_{8}=0.7744$ & $\hat{s}_{9}=0.8494$ & $\hat{s}_{10}=1.0460$ \\
$\hat{s}_{11}=1.1568$ & $\hat{s}_{12}=1.0271$ & & & \\
\hline
\end{tabular}

TABLE 3. Initialization of level, trend, seasonal and exponential smoothing parameters of the additive HW model for the e-commerce retail sales time series of Portugal.

\begin{tabular}{lcccc}
\hline Additive model & \multicolumn{3}{c}{ MSE $=452.3238$} \\
\hline$\hat{\alpha}=0.1765$ & $\hat{\beta}=0.0648$ & $\hat{\gamma}=0.6588$ & $\hat{l}_{1}=203.2920$ & $\hat{b}_{1}=2.2286$ \\
$\hat{s}_{1}=42.2531$ & $\hat{s}_{2}=13.6990$ & $\hat{s}_{3}=-15.7135$ & $\hat{s}_{4}=2.2865$ & $\hat{s}_{5}=25.6698$ \\
$\hat{s}_{6}=-14.9552$ & $\hat{s}_{7}=-21.7302$ & $\hat{s}_{8}=-47.7510$ & $\hat{s}_{9}=-32.2427$ & $\hat{s}_{10}=9.2656$ \\
$\hat{s}_{11}=33.6698$ & $\hat{s}_{12}=5.5490$ & & & \\
\hline
\end{tabular}

Table 4 shows the results of the accuracy measures calculated for training and testing periods for the two methods applied to the time series under study. As can be seen in the Table 4, according to the five measures of evaluation calculated, the multiplicative model is in fact the one with the best predictive (in the training period from January 2000 to December 2016) and forecast (in the testing period from January 2017 to February 2018) performances of the e-commerce retail sales time series of Portugal. Usually when a series displays a seasonal pattern characterized by an amplitude that varies with the series level, the multiplicative version is a better choice [4], as with the modeling process of this study.

TABLE 4. Forecasting performance evaluation of HW models of e-commerce retail sales time series of Portugal

\begin{tabular}{lcccccc}
\hline Model & MSE & RMSE & MAPE & MASE & U-Theil & \\
\hline Additive & 452.3238 & 21.2679 & 9.1284 & 0.7888 & 0.6311 & training period \\
Multiplicative & $\mathbf{4 2 0 . 3 2 3 7}$ & $\mathbf{2 0 . 5 0 1 8}$ & $\mathbf{8 . 4 6 9 7}$ & $\mathbf{0 . 7 4 0 5}$ & $\mathbf{0 . 5 8 6 6}$ & training period \\
\hline Additive & 298.9753 & 17.2909 & 17.9859 & 1.1051 & 1.0825 & testing period \\
Multiplicative & $\mathbf{2 6 5 . 2 4 2 6}$ & $\mathbf{1 6 . 2 8 6 3}$ & $\mathbf{1 6 . 7 6 2 8}$ & $\mathbf{1 . 0 2 9 9}$ & $\mathbf{1 . 0 2 1 5}$ & testing period \\
\hline
\end{tabular}

The mean square errors calculated from the 14-step-ahead required for the construction of the forecast intervals are summarized in Table 5. In Fig. 2 are represented the original values of the e-commerce time series, the estimates in the modeling period (training period), the forecasts in the forecasting period (testing period) and the forecast intervals for a confidence level of 95\%, applying the additive and the multiplicative model. The models' validation was assessed by means of the residuals analysis. The independency assumption was assessed by estimating the autocorrelation and the partial autocorrelation functions of residuals, by calculating the Durbin-Watson' statistics, and performing the Ljung-Box test and the assumption that the residuals are identically normally distributed were also verified (performing the Kolmogorov-Smirnov test).

It should be noted that the order of magnitude of these errors is big. In fact, this results in prediction intervals with large amplitudes, in terms of the coverage rate: the forecast intervals for the Holt-Winters model include the 
TABLE 5. Mean Square Error at $h$-step-ahead for the multiplicative HW model (the best performance) of ecommerce retail sales time series of Portugal.

\begin{tabular}{lccccccc}
\hline $\mathbf{h}$ & 1 month & 2 months & 3 months & 4 months & 5 months & 6 months & 7 months \\
$\mathbf{M S E}_{\mathbf{h}}$ & 420.3237 & 458.9360 & 470.3099 & 500.2939 & 515.3850 & 540.0650 & 616.2973 \\
\hline $\mathbf{h}$ & 8 months & 9 months & 10 months & 11 months & 12 months & 13 months & 14 months \\
$\mathbf{M S E}_{\mathbf{h}}$ & 681.5444 & 716.6324 & 749.2076 & 776.7510 & 819.6080 & 1111.9670 & 1141.6730 \\
\hline
\end{tabular}

(a) Additive

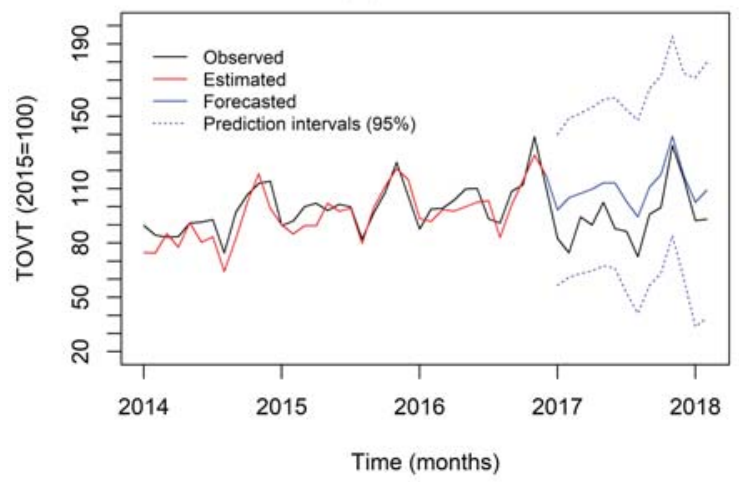

(b) Multiplicative

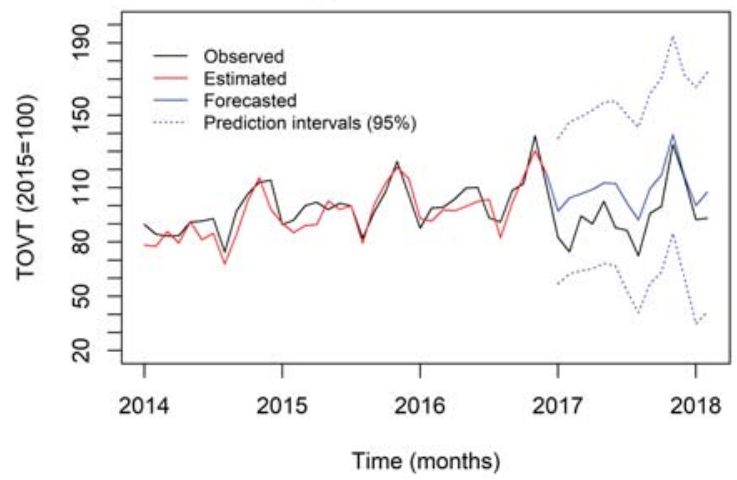

FIGURE 2. Additive HW (left) and multiplicative HW (right) estimates, forecasts, and forecast confidence intervals (95\%) for e-commerce retail sales time series of Portugal.

14 observations of the test time series, hence the coverage rate of $100 \%$ (see Fig. 2). However, the normality of prediction errors is, in this case, rejected at a significance level of 5\%, for all steps $h$, with $h=1,2, \ldots, 14$. Therefore, it is concluded that the prediction intervals constructed according to the expression (1) are not the most suitable for this type of data, i.e. the HW method is conservative in the construction of forecast intervals. In order to circumvent this failure, as future work an alternative is to study resampling methods for the construction of forecast intervals (an approach already considered in [1]).

\section{ACKNOWLEDGMENTS}

This research was partially financed by Portuguese funds by the Center for Research and Development in Mathematics and Applications (CIDMA) and the Portuguese Foundation for Science and Technology ("Fundação para a Ciência e a Tecnologia" - FCT), within project UID/MAT/04106/2019. This research was partially financed by Portuguese funds through Portuguese Foundation for Science and Technology ("Fundação para a Ciência e a Tecnologia" - FCT), within project UID/MAT/00013/2013.

\section{REFERENCES}

[1] M. Costa and A. M. Goncalves, "Forecasting time series combining holt-winters and bootstrap approaches," in Proceedings of the International Conference on Numerical Analysis and Applied Mathematics 2014 (ICNAAM-2014), AIP Conference Proceedings 1648 (T. E. Simos, G. Psihoyios and Ch. Tsitouras, Rhodes, Greece, 2015), pp. 110004-1-110004-4.

[2] P. R. Winters, Management Science 6, 324-342 (1960).

[3] RCoreTeam, R: a language and environment for statistical computing, https://www.R-project.org/ ( 2018).

[4] R. Hyndman and R. Snyder, Forecasting with Exponential Smoothing. The State Space Approach (Springer, 2008). 